\title{
Simulation of an all-optical photonic crystal switch using nonlinear optics and slow light for hetero structures y-junctions
}

\author{
Saeid Ghazanfari SHABANKAREH ${ }^{1}$, Rahim GHAYOUR ${ }^{2}$, Shahram Bahadori HAGHIGHI ${ }^{3}$, \\ Sajjad Ghazanfari SHABANKAREH ${ }^{4}$
}

${ }^{1}$ Department of Electrical and Computer Engineering, Azad Shiraz University, Shiraz, Iran

${ }^{2}$ Department of Electrical and Computer Engineering, Azad Shiraz University, Shiraz, Iran

${ }^{3}$ Department of Electrical and Computer Engineering, Shiraz University, Shiraz, Iran

${ }^{4}$ Department of Electrical and Computer Engineering, Azad Shiraz University, Shiraz, Iran

\begin{abstract}
In this paper we shows a photonic crystal mach-zender interferometer (MZI) based on new structure by coupled-cavity waveguide using slow light to shorten the length of waveguide arms and nonlinear properties of GaAs for design of an all optical switch. So we comparison with optical switches at arm's length that approximately is $10 \mu \mathrm{m}$. The design of $y$-juntion is performed using finite-difference time-domain method. finally the physical principals behind the phenomenon of slow light in photonic crystal waveguide is discussed.
\end{abstract}

Keywords: Photonic crystal, Mach-Zehnder interferometer, Hetero structure Y-junction, Band Gap, Slow light, Nonlinear optics.

\section{Introduction}

Photonic crystals (PhCs) due to their unique properties have attracted attention of many researchers. They believe $\mathrm{PhC}$ is a promising technology for the integrated optics. A revolution in the fields of optical signal processing and communication is hopefully not far from realization, like what happened to electronic circuits by semiconductor technology. In recent years, different types of optical devices using PhCs have been designed, simulated and even implemented [1-3].

Slow light phenomenon as an important key to control the flow of light and enhance the light-matter interactions can be attained in $\mathrm{PhC}$ waveguides. However, such a phenomenon is not observed in conventional ones in which the group velocity of guided modes $(v \mathrm{~g})$ is of the order of the velocity of light in vacuum (c) [4-6]. PhCs offer enhancement of nonlinear effects through the slow light phenomenon so that shrinkage of many optical devices such as modulators and switches becomes feasible [7-9]. As a matter of fact, the geometrical flexibility of $\mathrm{PhCs}$ is of the great interest to create suitable slow modes.

The keen interest in slow light in dielectrics is motivated by the fact that slow light adds functionality to a material by structuring alone. This is of great interest, as it allows to enhance the weak interaction in a material that may be of interest otherwise, such as silicon. Linear effects such as gain, thermo-optic and electro-optic interaction scale with the slowdown factor, whereas nonlinear effects may scale with its square [10, 11]. 
These two effects also represent the two limiting cases for slow light propagation in photonic crystal waveguides; the bandwidth is ultimately limited by the size of the Brillouin zone. In order to achieve a group velocity of $c / n g$, with $n g$ the group index, the maximum bandwidth can then be determined as follows :

$v_{g}=\frac{d w}{d k}=\frac{c}{n_{g}} \rightarrow \Delta v=\frac{1}{2 \pi} 0.5 \frac{2 \pi}{a}=\frac{c}{2 n_{g} a}$.

As an example, for $n \mathrm{~g}=100$ and a typical period of $a=0.4 \mu \mathrm{m}$ at $1.55 \mu \mathrm{m}$ wavelength, one obtains a bandwidth of $v=3.75 \mathrm{THz}$. In practice, and subject to good design and operation below the light line, it may be possible to achieve $20-30 \%$ of this bandwidth, which corresponds to around $1 \mathrm{THz}$.

Any type of optical switching device, linear or nonlinear, operates on the basis of a relative phase change, typically expressed as $\Delta k L$. In a Mach-Zehnder interferometer, for example, $\Delta k L=\pi$ for full on/off operation, whereas in a directional coupler, the condition is $\Delta k L=\sqrt{ } 3 \pi$. One usually assumes that $\Delta k$ can be expressed as $\Delta n k 0$, with $\Delta n$ the refractive index change induced by an external effect or by the nonlinearity of the material and $k 0$ the wave vector in vacuum [12-14].

Many optical modulators and switches are based on MZI in which the input light is split into two branches. During the propagation, light wave in one of the branches experiences a phase shift $(\Delta \phi)$ with respect to the other branch due to nonlinear effects .These two signals are combined at the output of MZI such that a constructive interference $(\Delta \phi=0)$ makes the switch ON and a destructive interference $(\Delta \phi=\pi)$ makes it OFF. Splitting (combining) light signal (signals) at the input (output) is done by use of a 3-dB directional coupler or a Yjunction $[15,16]$. Optical power splitters based on photonic-crystal Y-junctions have been investigated and optimized to enhance throughput efficiency and balance the distribution of power between output branches [17-19].Poor throughput efficiency in a $\mathrm{PhC}$ power splitter using square or triangular lattice is mainly due to back-reflections at the transition between different sections of the splitter. Optimization techniques are used to improve the performance of a beam splitter by minimizing backward reflections. Using hetero structure PhCs in which different $\mathrm{PhC}$ lattices are combined is an alternative approach to achieve optimal performance of $\mathrm{PhC}$ power splitters [20].

In this paper we introduce the physical principal slow light which shorten the arm's of wave guide and in section 2 we created a new y-junction hetero structure with start a new unit cell which previous well-known type of ccw. In section 3 we suggest a new hetero structure and improve its through put efficiency. Improvement is done step by step through a theoretical approach and adjusting the radii of some rods near the junctions between the different sections of the optical splitter [21].finally in section 4 we show Refractive index with the change in the output switch is off and return signal. 


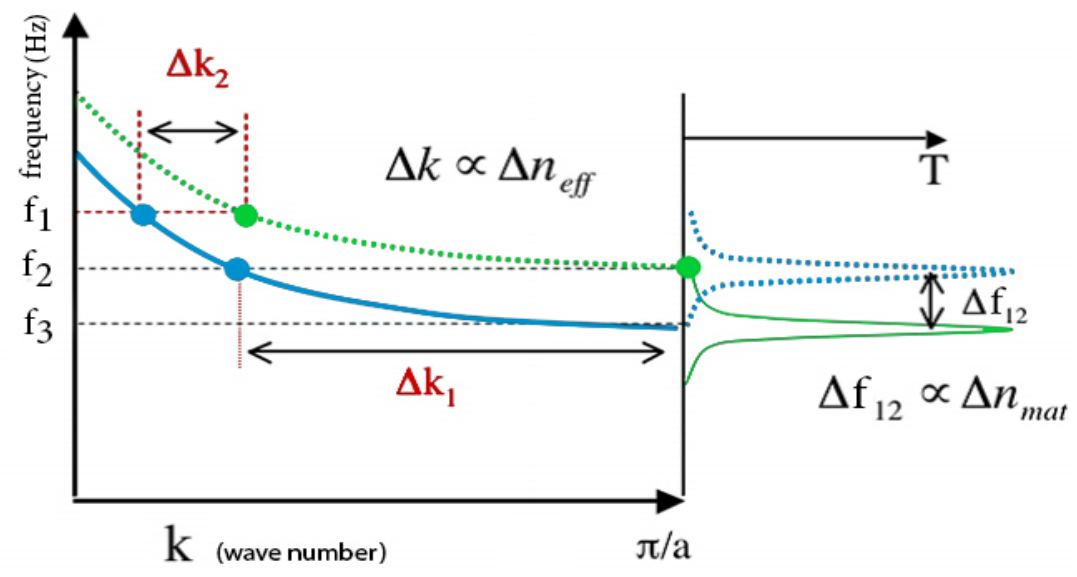

Fig. 1: Illustration of the difference between the effect of the material index $n_{\text {mat }}$ and the effective modal index $n_{\text {eff }}$ in the slow light regime.

The dispersion curve of a given mode is described by the solid line. Since a mode represents a resonance condition of the waveguide, it can be depicted as a transmission peak, indicated to the right. If the material index is changed, the resonance moves accordingly, and the corresponding change in resonance frequency is proportional to the change in index, so $\Delta \omega / \omega \approx \Delta n / n \Rightarrow \Delta \omega 12 \propto \Delta n$ mat, where $\Delta \mathrm{w}=2 \pi \Delta \mathrm{f}$, This is indicated by the dotted line. The change experienced by the guided mode is different, however. It is given by the change in effective modal index and can be derived from the wave equation as follows:

$c=\omega / k \Leftrightarrow c 0 / n$ eff $=\omega / k \Rightarrow \Delta n$ eff $=(c 0 / \omega) \Delta k$, with $c 0$ the speed of light in vacuum. It is immediately obvious from figure 1 that where the dispersion curve is flat, i.e in the slow light regime, the $\Delta k$ and therefore the $\Delta n$ eff is much larger than in the fast light regime where the dispersion curve is steeper, hence $\Delta k 1>\Delta k 2$. So a slow mode experiences a larger change in effective index than a fast mode, despite the fact that the change in material index $\Delta n$ mat is the same in both the cases. Since $\Delta k$ scales with the slope of the dispersion curve, it also scales with the slowdown factor, which allows us to write the condition for switching as $\Delta k L$ $=\pi,(2)[22]$.

\section{Photonic crystal with new ccw.}

The schematic of a new (phc) ccw unit cell, is shown in figure 2.

a

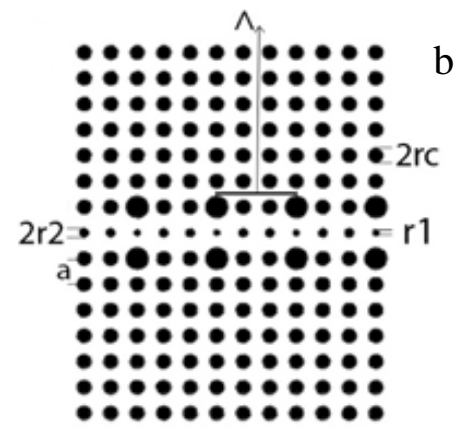

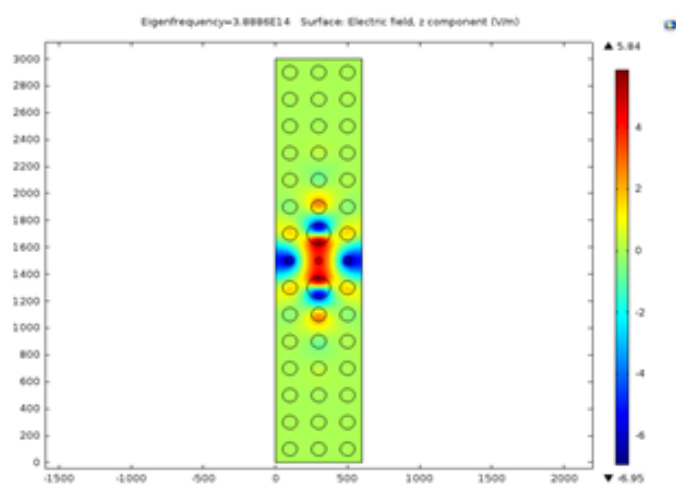

Fig.2.a) the schematic of new square lattice ccw .b)electric field 
The structure is based on square lattice Phc with refractive index GaAs=3.5031, figure 2. The structure is the radius of the hole $\Lambda=3 \mathrm{a}, \mathrm{r} 1=0.1 \mathrm{a}, \mathrm{r} 2=0.15 \mathrm{a}, \mathrm{rc}=0.25 \mathrm{a}$, the band structure of new $\mathrm{ccw}$ is calculated by finite-element method (FEM) that been done Comsol for TE polarization ,as you can see 7 guided mode with in the photonic band gap between $\mathrm{fl} \approx 3.9 \mathrm{e} 14$ to $\mathrm{f} 7 \approx 5.1 \mathrm{e} 14$ in figure 3 is shown. Only guided modes $1,5,6$ do not overlap with the other modes , Therefore, one of the three modes has to be selected as the propagating mode to avoid intermodal dispersion. The electric-field pattern associated with the guided mode 7 in a super cell of the structure is shown in figure $2 b$.

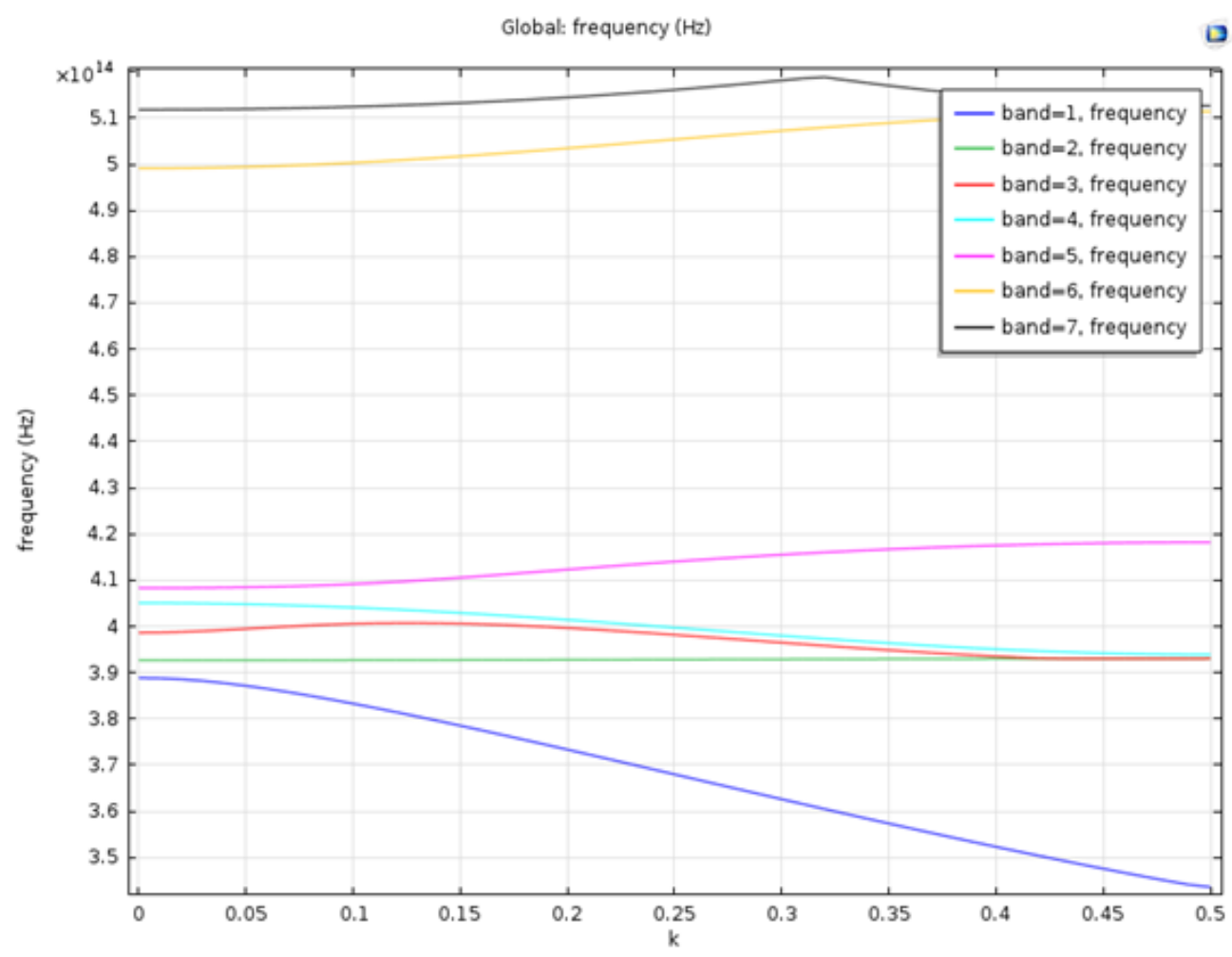

Fig. 3: The corresponding band structure

Let's talk about the advantages of this unit cell where slow light is better than the previous type is evident in figure $4 \mathrm{a}, \mathrm{b}$. the structure of adjacent cavity by as much as 0.6
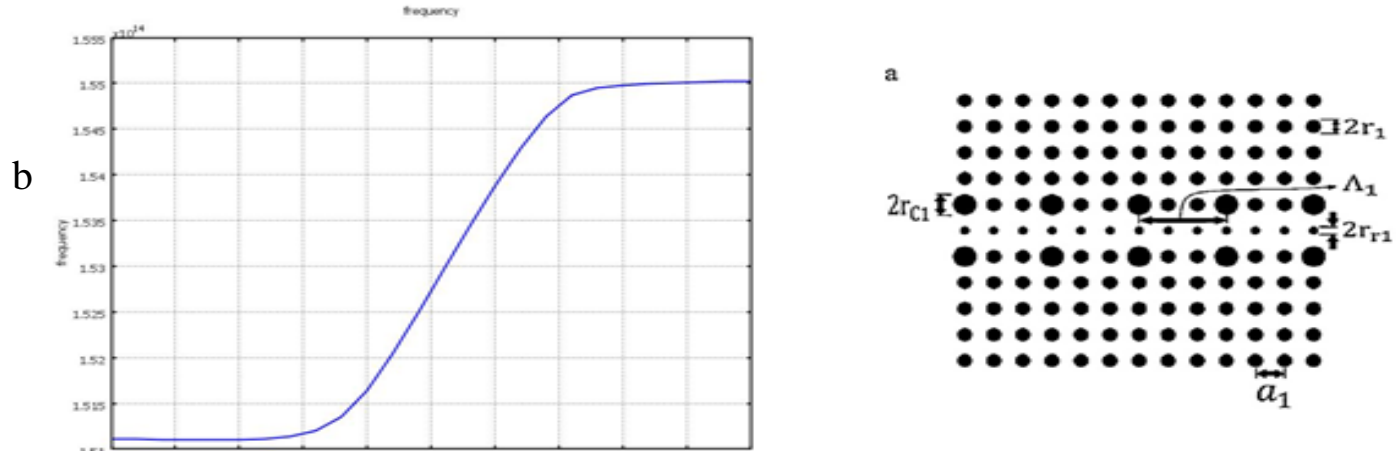

Fig: 4(a) The schematic of Previous lattice. (b) Band structure of previous unit cell. radius changed and as well as the structure of a row at the top and bottom bars have added GaAs, which is to improve the slow light ,in figure 4c. It is well recognized that there is an 
intrinsic compromise between the slow light phenomenon and the available band width. In other words, achieving slow modes comes at the price of the shrinkage of the bandwidth. On the other side, it has been proven that more physical distance between the cavities (greater $\Lambda$ ) makes the group velocity of the guided mode slower [23].

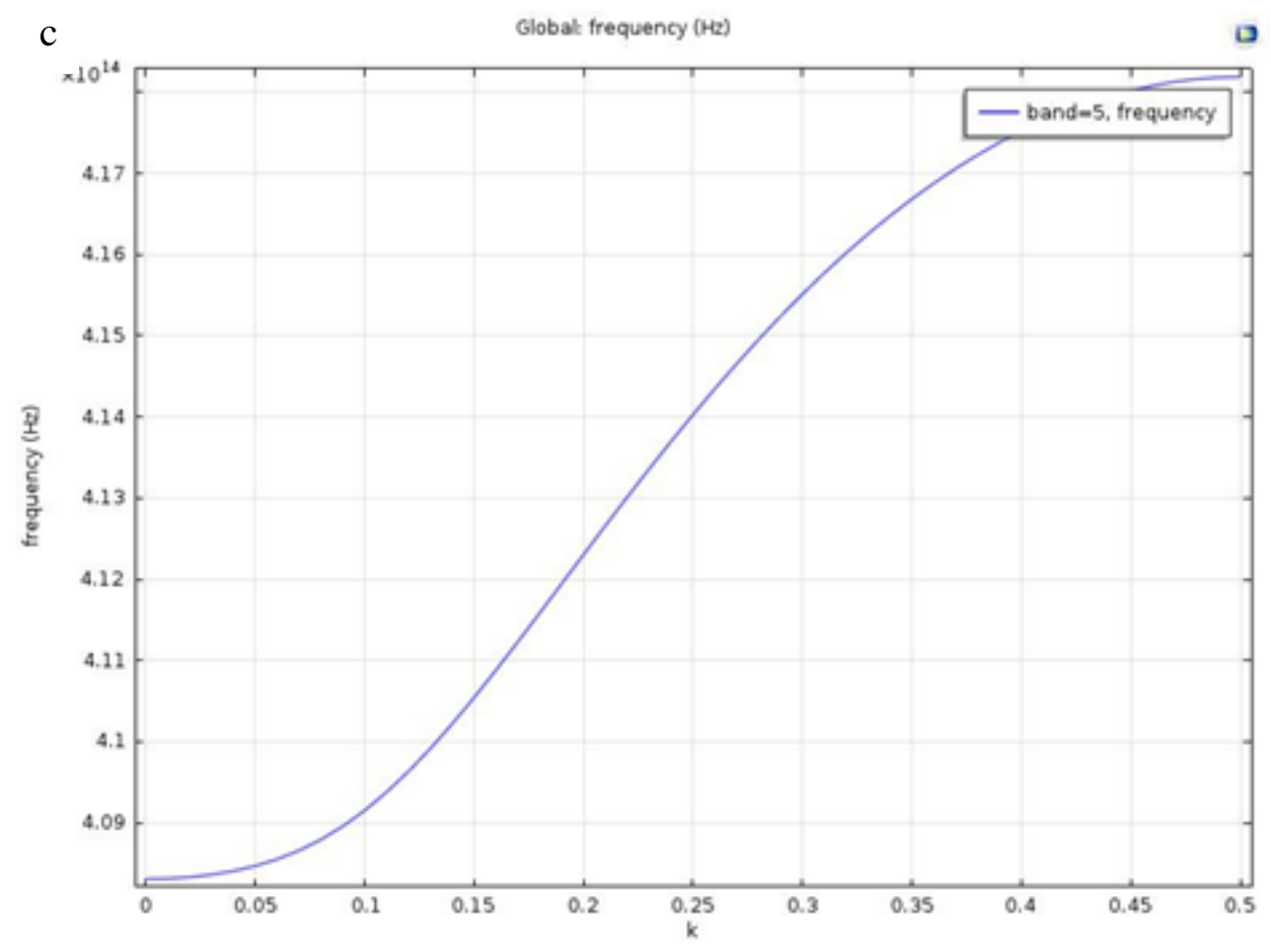

Fig. 4c: The band structure of new unit cell

\section{Design of hetero structure $y$-junction of new $\mathrm{ccw}$.}

Shows the schematic diagram of the PhC MZI designed in this work. As shown at the top of the figure, CCW1 is used as the arms of the Mach-Zehnder structure. Different sections of the structure are also specified in Fig. 5. The Y-junctions which are used at the input and output of the MZI act as a beam splitter and combiner, respectively. The Y-splitter consists of a wave guiding section (section A) and a wave-splitting section (section B). section B is also divided into two parts: (i)the slanted waveguide subsection (subsection B1) and (ii) the horizontal waveguide subsection (subsections B2). By an ideal optical splitter, dividing the input optical power into two output ports of the splitter equally and without significant losses is expected. How-ever, back-reflections at the transition between the wave guiding and wavesplitting sections and also the bending losses at the corner joints between subsections B1 and B2 degrade the performance of the splitter notably. In order to reduce the above mentioned losses, the $\mathrm{PhC}$ waveguides which are used in all the sections have tube of the same type. By doing this, we try to minimize mismatches between different sections of the splitter, Therefore, PhC CCW1 has to be used in the wave guiding section. However, it has been proved that a square lattice is not suited for the slanted waveguide subsection (subsection B1) [23]. 


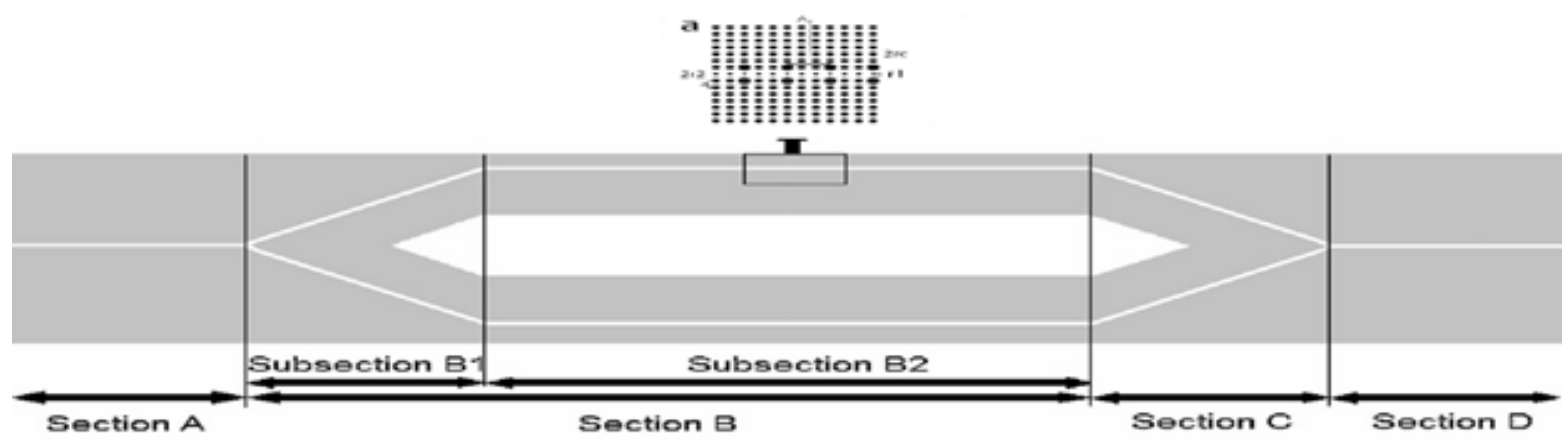

Fig 5: Sketch of the PhC Mach-Zehnder interferometer with the structure of CCW1used in its arms as displayed at the top of this figure. Different sections of the structure are illustrated.

\section{Show in frequency domain in MZI.}

As you seen when we change the refractive index of 5 cavity in end of the beam splitter in MZI which is contact with output, we see that of output is return and its off and when the refractive index no change, the input appears in output, we see in figure6.a. The output signal and the input mode on and off to see in figure6.b.

a

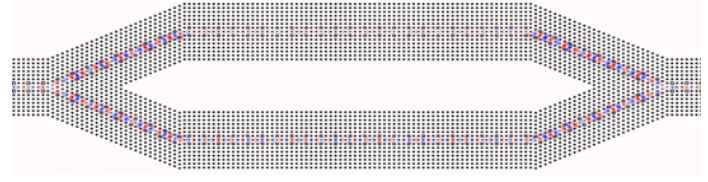

$\mathrm{b}$

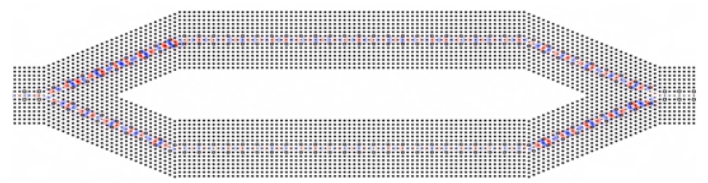

Fig 6a: Snapshots of a Gaussian pulse propagating down the Mach-Zehnder inter-ferometer wave guide. 
a
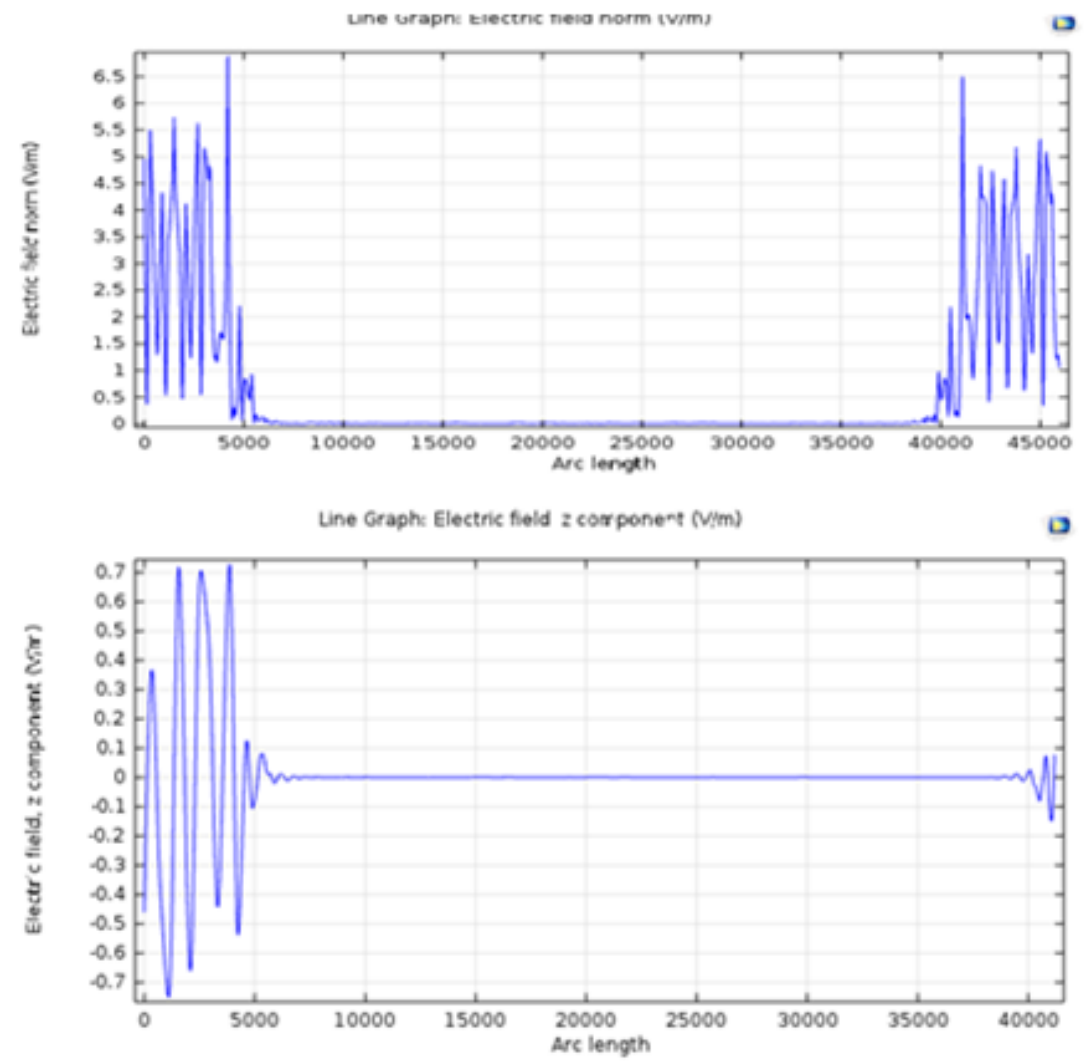

Fig.7: (a) switch is on and (b) the switch is off.

\section{Group velocity of the new ccw.}

Since the group velocity of a waveguide mode defines the speed at which the optical energy transmits through that mode, we can conclude that the decay rates of the cavity mode into the waveguides are proportional to the group velocities. We see the group velocity in figure 7 that is approximately 60.

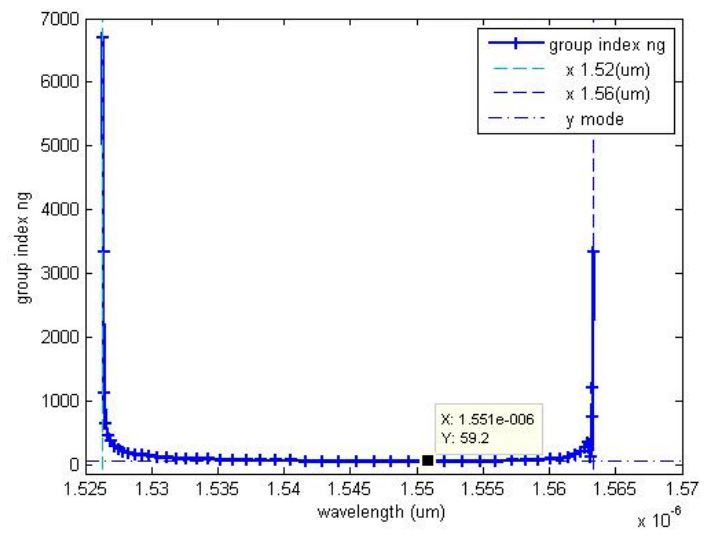

Fig. 8: Group velocity of the new ccw. 


\section{Conclusion}

In summary, we demonstrated that the new $\mathrm{CCW}$ provides the possibility of achieving slow modes by modifying the radii of the cavity rods rather than increasing the physical distance between the cavities which limits the available bandwidth considerably. Therefore, a MZI using the new CCW was proposed for the purposes of optical switching. In order to realize the structure of the MZI , a hetero structure Y-junction consisting of square and hexagonal lattices of the new $\mathrm{CCW}$ was designed based on temporal coupled-mode theory and optimized in two steps. We also according to equation 1 in about 7.1 terahertz of bandwidth which operates 30 per cent of it is that you $2.1 \mathrm{THz}$. As a result, an overall throughput efficiency of more than $90 \%$ was attained. Then, each arm of the MZI was determined to be as short as $\mu \mathrm{m}$. Finally, the transient simulation of the proposed photonic crystal MZI as a good candidate for optical integration was performed and the results were shown and discussed.

\section{References}

[1] W. Jiang, L. Gu, X. Chen, R.T. Chen, Photonic crystal waveguide modulators for silicon photonics: device physics and some recent progress, Solid State Electron. 51 (2007) 1278-1286.

[2] A. Esmaieli, R. Ghayour, Magneto-optical photonic crystal $1 \times 3$ switchable power divider, Photon. Nanostruct. Fundam. Appl. 10 (2012) 131-139.

[3] A. Taher Rahmati, N. Granpayeh, Kerr nonlinear switch base on ultra-compact photonic crystal directional coupler, Optik 122 (2011) 502-505.

[4] M. Solja ci'c, J.D. Joannopoulos, Enhancement of nonlinear effects using photonic crystals, Nat. Mater. 3 (2004) 211-219.

[5] T.F. krauss, Slow light in photonic crystal waveguides, J. Phys. D: Appl. Phys. 40(2007) 2666-2670.

[6] T. Baba, Slow light in photonic crystals, Nat. Photon. 2 (2008) 465-473.

[7] C. Monat, B. Corcoran, D. Pudo, M. Ebnali, C. Grillet, M.D. Pelusi, D.J. Moss, B.J.Eggleton, T.P. White, L. O'Faolain, T.F. Krauss, Slow light enhanced nonlinear optics in silicon engineered photonics crystal waveguides, IEEE J. Sel. Topics Quantum Electron. 16 (2010) 344-356.

[8] Y. Jiang, W. Jiang, L. Gu, X. Chen, R.T. Chen, 80-micron interaction length silicon photonic crystal waveguide modulator, Appl. Phys. Lett. 87 (2005) 221105.

[9] L. Gu, W. Jiang, X. Chen, R.T. Chen, mechanism of p-i-n diode-based pho-tonic crystal silicon electro optic modulators for gigahertz operation, IEEE J.Sel. Topics Quantum Electron. 14 (2008) 1132-1139.

[9] Soljacic M and Joannopoulos J D 2004 Nature Mater.3 211

[10] Soljacic M, Johnson S G, Fan S, Ibanescu M, Ippen E and Joannopoulos J D 2002 J. Opt. Soc. Am. B 19 2052

[14] Petrov A Y and Eich M 2004 Appl. Phys. Lett.85 4866

[15] Frandsen L H, Lavrinenko A V, Fage-Pedersen J and Borel P I 2006 Opt. Express 149444

[16] Biallo D, Ayre M, Karle T J, Settle M, D’Orazio A and Krauss T F 2007 at press

[15] T. Fujisawa, M. Koshiba, Finite-element modeling of nonlinear Mach-Zehnder interferometers based on photonic-crystal waveguides for all-optical signal processing, J. Light wave Technol. 24 (2006) 617-623.

[16] E. Cassan, X. Le Roux, C. Caer, R. Hao, D. Bernier, D. Maris-Morini, L. Vivien,Silicon slow light photonic crystal structures: present achievements and future trends, Front. Opto electron. China 4 (2011) 243-253. 
[17] R. Wilson, T.J. Karle, I. Moerman, T.F. Krauss, Efficient photonic crystal Y-junctions, J. Opt. A: Pure Appl. Opt. 5 (2003) S76-S80.

[18] L.H. Frandsen, P.I. Borel, Y.X. Zhuang, A. Harpøth, M. Thorhauge, M. Kris-tensen, W. Bogaerts, P. Dumon, R. Baets, V. Wiaux, J. Wouters, S. Beckx,Ultralow-loss 3-dB photonic crystal waveguide splitter, Opt. Lett. 29 (2004)1623-1625.

[19] S. Li, H.-W. Zhang, Q.-Y. Wen, F.-M. Bai, W.-W. Ling, Y.-S. Xie, Y.-X. Li, Improved Y-splitter photonic crystal waveguides in terahertz regime, Appl. Phys. B: LasersOpt. 99 (2010) 709-716.

[20] A. Sharkawy, S. Shi, D.W. Prather, Hetero structure photonic crystals: theory and applications, Appl. Opt. 41 (2002) 7245-7253.

[21] Photonic crystal optical switch using a new slow light waveguide and hetero structure Y-junctions Shahram Bahadori Haghighi, Rahim Ghayour*, Babak Vakili , Optik 124 (2013) 6292- 6297.

[22] T.F. krauss, Slow light in photonic crystal waveguides, J. Phys. D: Appl. Phys. 40(2007) 2666-2670.

[23] Bahadori,S., Ghayour,R., Optical self-phase modulation using a new photonic crystal coupled-cavity waveguide. Optica Applicata, Vol. XLIV, No. 1, (2014). 